\title{
Two More Symmetric Properties of Odd Numbers
}

\author{
Xingbo WANG \\ (Department of Mechatronics, Foshan University, Foshan City, Guangdong Province, PRC, 528000)
}

\begin{abstract}
The article proves another two new symmetric properties of odd numbers. The new properties are helpful to know odd numbers in a deep level, especially in knowing the distribution of an odd number's multiples and divisors.
\end{abstract}

Keywords : odd numbers, symmetric property, multiple numbers, divisors

\section{Introduction}

Article [1] put forward the concept of valuated binary tree and declared it to be a new approach to study integers. Article [2] continued listing several new properties of old integers based on the valuated tree, including symmetric property of nodes, symmetric common divisor between root-node and its descendant nodes. Based on the two articles, articles [3] discovered genetic traits of odd numbers and proposed a method to factorize odd numbers. Look into the three articles, it is can see that the symmetry plays key role in the whole valuated binary tree. This article continues revealing two more symmetric properties of odd numbers.

\section{Definitions, Notations and Lemmas}

Let $N_{(0,0)} \geq 3$ be an odd number; the $N_{(0,0)}$-rooted binary tree, denoted by $T_{N_{(0,0)}}$ is defined in [1]. This article continues using the symbols and notations that were used in [1]. The following lemmas are from [1] and [2].

Lemma 1(See in [1]). Let $\boldsymbol{T}$ be a recursively-constructed $N_{(0,0)}$-rooted binary tree; then the following statements hold

(1) There are $2^{k}$ nodes on the $k^{\text {th }}$ level, $k=0,1, \ldots$;

(2) Node $N_{(k, j)}$ is computed by

$$
\begin{aligned}
& N_{(k, j)}=2^{k} N_{(0,0)}-2^{k}+2 j+1 \\
& k=0,1,2, \ldots ; j=0,1, \ldots, 2^{k}-1
\end{aligned}
$$

(3) The two nodes, $N_{(i, \omega)}$ and $N_{\left(i, 2^{i}-1-\omega\right)}$, are at the symmetric position of the $i^{\text {th }}$ level and they satisfy

$$
N_{(i, \omega)}+N_{\left(i, i^{i}-1-\omega\right)}=2^{i+1} N_{(0,0)}
$$

Lemma 2 (See in [2]). Suppose node $N_{(0,0)}$ has a common divisor $d$ with $N_{(i, \omega)}$, then $d$ is also a common divisor of $N_{(0,0)}$ and $N_{\left(i, 2^{i}-1-\omega\right)}$.

Lemma 3 (See in [1]). Let $p$ be a positive odd integer; then among $p$ consecutive positive odd integers there exists one and only one that can be divisible by $p$.

Lemma 4. For arbitrary integer $\alpha \geq 1$, it holds

$$
2^{2 \alpha-1}+1 \equiv 0(\bmod 3) \text { and } 2^{2 \alpha-2}-1 \equiv 0(\bmod 3)
$$

Proof. Proof by mathematical deduction. Obviously, the conclusion holds for $\alpha=1$ because $2^{2 \times 1-1}+1=3 \equiv 0(\bmod 3)$ and $2^{2 \times 1-2}-1=0 \equiv 0(\bmod 3)$.

Now assume the conclusion holds for $\alpha=m$, namely, $2^{2 m-1}+1 \equiv 0(\bmod 3)$; then it yields

$$
4 \times\left(2^{2 m-1}+1\right)=2^{2 m+1}+4 \equiv 2^{2(m+1)-1}+1 \equiv 0(\bmod 3)
$$

Note that $2^{2 \alpha-2}-1=\left(2^{\alpha-1}-1\right)\left(2^{\alpha-1}+1\right)$ and the remainder of $2^{x}$ divided by 3 must be 1 or 2 for arbitrary integer $x \geq 0$, it knows $2^{2 \alpha-2}-1=\left(2^{\alpha-1}-1\right)\left(2^{\alpha-1}+1\right) \equiv 0(\bmod 3)$ always holds. 
Lemma 5. For arbitrary integer $\alpha \geq 1$, it holds

$$
2^{2 \alpha-1}-1 \neq 0(\bmod 3) \text { and } 2^{2 \alpha-2}+1 \neq 0(\bmod 3)
$$

Proof. By Lemma 4, it knows that the remainder of $2^{2 \alpha-1}$ divided by 3 is 2 and the remainder of $2^{2 \alpha-2}$ divided by 3 is 1 for arbitrary integer $\alpha \geq 1$. Hence the lemma holds.

\section{New Results and Proofs}

Theorem 1. Let $N_{(0,0)}$ be an odd number bigger than 1 and $T_{N_{(0,0)}}$ be the $N_{(0,0)}$-rooted binary tree; if node $N_{(i, \omega)}$ has a common divisor $d$ with node $N_{\left(i, 2^{i}-1-\omega\right)}$, then $d$ is also a divisor of $N_{(0,0)}$.

Proof. Let $N_{(i, \omega)}=d a$ and $N_{\left(i, i^{i}-1-\omega\right)}=d b$; since $N_{(i, \omega)}$ and $N_{\left(i, 2^{i}-1-\omega\right)}$ are both odd numbers, it knows that $d$ is also an odd number. Then by (2) it yields

$$
d a+d b=2^{i+1} N_{(0,0)}
$$

and then it holds

$$
d \mid N_{(0,0)}
$$

Theorem 2. Let $N>1$ be odd number, $3 \leq p \leq N$ be an odd prime and $k>1$ be integer; suppose $a_{i}=2^{k}(N-1)+2 i+1, i=0,1, \ldots, 2^{k}-1$; then the following conclusions hold

(1) If $p \nmid N$, then $p \mid a_{\omega} \Rightarrow p \nmid a_{2^{k}-1-\omega}$ for arbitrary $0 \leq \omega \leq 2^{k}-1$.

(2) If $p \mid N$, then $p\left|a_{\omega} \Rightarrow p\right| a_{2^{k}-1-\omega}$ for arbitrary $0 \leq \omega \leq 2^{k}-1$.

Proof. Since the case $p \mid N$ is just the case of Lemma xx, here only prove the case $p \nmid N$.

Let $N=p s+r, 1 \leq r \leq p-1$; then

$$
a_{\omega}=2^{k}(p s+r-1)+2 \omega+1=2^{k} p s+2^{k} r-\left(2^{k}-2 \omega-1\right)
$$

Thus $p \mid a_{\omega}$ yields $p \mid\left(2^{k} r-\left(2^{k}-2 \omega-1\right)\right)$, namely, $2^{k} r-\left(2^{k}-2 \omega-1\right)=p q$ where $q \geq 1$ is integer;

then

Note that

$$
2^{k}-2 \omega-1=2^{k} r-p q
$$

$$
\begin{aligned}
& a_{2^{k}-1-\omega}=2^{k}(p s+r-1)+2\left(2^{k}-1-\omega\right)+1 \\
& =2^{k} p s+2^{k} r-2^{k}+2^{k+1}-2-2 \omega+1 \\
& =2^{k} p s+2^{k} r+2^{k}-2 \omega-1
\end{aligned}
$$

Substituting $2^{k}-2 \omega-1$ in (4) by $2^{k} r-p q$ yields

$$
\begin{aligned}
& a_{2^{k}-1-\omega}=2^{k} p s+2^{k} r+2^{k}-2 \omega-1 \\
& =2^{k} p s+2^{k} r+2^{k} r-p q \\
& =2^{k} p s-p q+2^{k+1} r
\end{aligned}
$$

It immediately knows that $p \nmid a_{2^{k}-1-\omega}$ because $p \nmid 2^{k+1} r$.

Particularly, when $p=3$ it leads to the following Corollary 1 .

Corollary 1. Let $N>1$ be odd number and $k>1$ be integer; suppose $a_{i}=2^{k}(N-1)+2 i+1$, $i=0,1, \ldots, 2^{k}-1$; then $3 \nmid N$ and $3 \mid a_{\omega}$ yield $3 \nmid a_{2^{k}-1-\omega}$ for arbitrary $0 \leq \omega \leq 2^{k}-1 ; 3 \mid N$ and $3 \mid a_{\omega}$ yield $3 \mid a_{2^{k}-1-\omega}$ for arbitrary $0 \leq \omega \leq 2^{k}-1$.

Theorem 3. Suppose $m>3$ is an integer and $N=p q$ is an odd number, where $\mathrm{p}$ and $\mathrm{q}$ are prime numbers bigger than 3; Let $e_{m}^{p}=2^{m} N-p, e_{m}^{p+1}=e_{m}^{p}+2$ and $e_{m}^{p-1}=e_{m}^{p}-2$; then there are the divisible relations as shown in the following table. 


\begin{tabular}{|c|c|c|c|}
\hline$N$ & $p$ & $m$ is odd & $m$ is even \\
\hline \multirow{2}{*}{$N=3 n+1$} & $p=3 \mathrm{~s}+1$ & $3 \nmid\left(e_{m}^{p}-2\right), 3 \nmid e_{m}^{p}, 3 \mid\left(e_{m}^{p}+2\right)$ & $3 \nmid\left(e_{m}^{p}-2\right), 3 \mid e_{m}^{p}, 3 \nmid\left(e_{m}^{p}+2\right)$ \\
\cline { 2 - 5 } & $p=3 s+2$ & $3 \nmid\left(e_{m}^{p}-2\right), 3 \mid e_{m}^{p}, 3 \nmid\left(e_{m}^{p}+2\right)$ & $3 \mid\left(e_{m}^{p}-2\right), 3 \nmid e_{m}^{p}, 3 \nmid\left(e_{m}^{p}+2\right)$ \\
\hline \multirow{2}{*}{$N=3 n+2$} & $p=3 s+1$ & $3 \nmid\left(e_{m}^{p}-2\right), 3 \mid e_{m}^{p}, 3 \nmid\left(e_{m}^{p}+2\right)$ & $3 \nmid\left(e_{m}^{p}-2\right), 3 \nmid e_{m}^{p}, 3 \mid\left(e_{m}^{p}+2\right)$ \\
\cline { 2 - 4 } & $p=3 \mathrm{~s}+2$ & $3 \mid\left(e_{m}^{p}-2\right), 3 \nmid e_{m}^{p}, 3 \nmid\left(e_{m}^{p}+2\right)$ & $3 \nmid\left(e_{m}^{p}-2\right), 3 \mid e_{m}^{p}, 3 \nmid\left(e_{m}^{p}+2\right)$ \\
\hline
\end{tabular}

Proof. Since $N$ is not a 3's multiple, it takes the form of either $3 n+1$ or $3 n+2$. Since $p$ can take the form of either $3 s+1$ or $3 s+2$, the following deductions validate the conclusion.

$$
\begin{aligned}
& \left\{\begin{array}{c}
N=3 n+1 \\
p=3 s+1
\end{array} \Rightarrow e_{m}^{p}=2^{m}(3 n+1)-3 s-1=2^{m} \times 3 \times n-3 s+2^{m}-1\right. \\
& \left\{\begin{array}{l}
N=3 n+1 \\
p=3 s+1
\end{array} \Rightarrow e_{m}^{p}+2=2^{m} \times 3 \times n-3 s+2^{m}+1\right. \\
& \left\{\begin{array}{l}
N=3 n+1 \\
p=3 s+1
\end{array} \Rightarrow e_{m}^{p}-2=2^{m} \times 3 \times n-3(s+1)+2^{m}\right. \\
& \left\{\begin{array}{l}
N=3 n+1 \\
p=3 s+2
\end{array} \Rightarrow e_{m}^{p}=2^{m}(3 n+1)-3 s-2=2^{m} \times 3 \times n-3(s+1)+2^{m}+1\right. \\
& \left\{\begin{array}{l}
N=3 n+1 \\
p=3 s+2
\end{array} \Rightarrow e_{m}^{p}+2=2^{m}(3 n+1)-3 s+2^{m}\right. \\
& \left\{\begin{array}{l}
N=3 n+1 \\
p=3 s+2
\end{array} \Rightarrow e_{m}^{p}-2=2^{m} \times 3 \times n-3(s+1)+2^{m}-1\right. \\
& \left\{\begin{array}{c}
N=3 n+2 \\
p=3 s+1
\end{array} \Rightarrow e_{m}^{p}=2^{m}(3 n+2)-3 s-1=2^{m} \times 3 \times n-3 s+2^{m+1}-1\right. \\
& \left\{\begin{array}{c}
N=3 n+2 \\
p=3 s+1
\end{array} \Rightarrow e_{m}^{p}+2=2^{m} \times 3 \times n-3 s+2^{m+1}+1\right. \\
& \left\{\begin{array}{c}
N=3 n+2 \\
p=3 s+1
\end{array} \Rightarrow e_{m}^{p}-2=2^{m} \times 3 \times n-3(s+1)+2^{m+1}\right. \\
& \left\{\begin{array}{c}
N=3 n+2 \\
p=3 s+2
\end{array} \Rightarrow e_{m}^{p}=2^{m}(3 n+2)-3 s-2=2^{m} \times 3 \times n-3(s+1)+2^{m+1}+1\right. \\
& \left\{\begin{array}{l}
N=3 n+2 \\
p=3 s+2
\end{array} \Rightarrow e_{m}^{p}+2=2^{m} \times 3 \times n-3 s+2^{m+1}\right. \\
& \left\{\begin{array}{c}
N=3 n+2 \\
p=3 s+2
\end{array} \Rightarrow e_{m}^{p}-2=2^{m} \times 3 \times n-3(s+1)+2^{m+1}-1\right.
\end{aligned}
$$

Referring to Lemmas 4 and 5, the theorem holds.

\section{Conclusion}

Theorem 1 in deeded reveals a new trait of odd numbers' symmetry. In fact, Theorem 1 and Lemma 2 form a close chain to disclose the relationships between the root and its descendant notes in a valuated binary tree. Theorem 2 exhibits an asymmetric relation. This asymmetry actually essentially shows another side of odd numbers' symmetry. Theorem 3 is actually an instance of Corollary 1 and a very useful reference for analyzing distribution of $e_{m}^{p}=2^{m} N-p$ that has a common divisor with odd composite number $N=p q$. By Theorem 3, it knows that half of $e_{m}^{p}$ occur in 3's multiples. This might be helpful for designing a faster search program when factoring $N$ by means of finding the greatest common divisor between $N$ and $e_{m}^{p}$. Hope to see the following researches in this aspect. 


\section{Acknowledgements}

The research work is supported by Department of Guangdong Science and Technology under projects 2015A030401105 and 2015A010104011, Foshan Bureau of Science and Technology under projects 2016AG100311, and projects 2014SFKC30 and 2014QTLXXM42 from Guangdong Education Department. The authors sincerely present thanks to them all.

\section{References}

[1] WANG Xingbo, Valuated Binary Tree: A New Approach in Study of Integers, International Journal of Scientific and Innovative Mathematical Research (IJSIMR), 4(3), 2016, 63-67

[2] Xingbo WANG, Amusing Properties of Odd Numbers Derived From Valuated Binary Tree, IOSR Journal of Mathematics, 12( 6,Ver.V),2016,53-57

[3] Xingbo WANG, Genetic Traits of Odd Numbers With Applications in Factorization of Integers, Global Journal of Pure and Applied Mathematics,13(1),2017,318-333 